\title{
Homoclinic and non-wandering points for maps of the circle
}

\author{
LOUIS BLOCK, ETHAN COVEN, IRENE MULVEY \\ AND ZBIGNIEW NITECKI
}

\begin{abstract}
Department of Mathematics, University of Florida, Gainesville, FL 32611, USA; Department of Mathematics, Wesleyan University, Middleton, CT 06457, USA; Department of Mathematics, Swarthmore College, Swarthmore, PA 19081, USA; Department of Mathematics, Tufts University, Medford, MA 02155, USA
\end{abstract}

(Received 4 January 1983 and revised 15 May 1983)

\begin{abstract}
For continuous maps $f$ of the circle to itself, we show: (A) the set of nonwandering points of $f$ coincides with that of $f^{n}$ for every odd $n$; (B) $f$ has a horseshoe if and only if it has a non-wandering homoclinic point; $(\mathrm{C})$ if the set of periodic points is closed and non-empty, then every non-wandering point is periodic.
\end{abstract}

\section{Introduction}

In this paper we examine the dynamics of continuous maps of the circle to itself, establishing for such maps versions of three results known to hold for maps of a compact interval.

THEOREM A. If $f$ is a continuous map of the circle, then the set of non-wandering points of $f$ coincides with that of $f^{n}$ for every odd $n$.

Theorem $A$ is identical to the corresponding result [3] for maps of the interval.

THEOREM B. A continuous map of the circle has a horseshoe if and only if it has a non-wandering homoclinic point.

Here we have added to the corresponding result [1], [9], [10] for maps of the interval the condition that the homoclinic point is non-wandering. We show by example that on the circle this condition cannot be omitted. Actually we prove a stronger result - see theorem $\mathrm{B}+$ and the remarks following it at the end of $\S 5$.

THEOREM C. If the set of periodic points of a continuous map of the circle is closed and non-empty, then every non-wandering point is periodic.

Here we have added to the corresponding result $[4],[8],[11]$ for maps of the interval the obvious requirement that the set of periodic points is non-empty - consider an irrational rotation.

We will prove theorem A by adapting the proof in [3] to the circle. In fact we will produce a shorter proof, valid for the interval as well as the circle. We will prove theorems $\mathrm{B}$ and $\mathrm{C}$ by lifting the map of the circle to a map of the reals, for which these results are known to hold. We will then project back down to the circle. 


\section{Preliminaries}

Throughout this paper $f$ will denote a continuous map of the circle to itself. The set of non-wandering points of $f$ will be denoted by $\Omega(f)$ and the set of periodic points by $\operatorname{Per}(f) . \Omega(f)$ is always closed and non-empty and

holds for all $n$.

$$
\operatorname{Per}(f)=\operatorname{Per}\left(f^{n}\right) \subseteq \Omega\left(f^{n}\right) \subseteq \Omega(f)
$$

Formally we will think of the circle as $\mathbb{R} / \mathbb{Z}$ and use $\pi$ to denote the canonical projection. Thus every continuous map $f$ of the circle has countably many lifts, i.e. continuous maps $F: \mathbb{R} \rightarrow \mathbb{R}$ satisfying

$$
f \circ \pi=\pi \circ F \text {. }
$$

Any two such lifts differ by an integer and the unique integer $d$ satisfying

$$
F(X+1)=F(X)+d
$$

for all lifts $F$ and all $X$ is called the degree of $f$, denoted $\operatorname{deg}(f)$.

In addition, we will think of the circle as oriented so that $\pi$ is orientationpreserving. Thus notation such as $[a, b]$ will make sense on the circle as well as on the interval or reals. In all three cases, we will have occasion to refer to points near a given point as being on the positive $(+)$ or negative $(-)$ side of that point. When we wish to speak of a side without specifying which it is we will use the letter $S$.

We define a (basic) half-neighbourhood of a point to be a non-degenerate closed interval having that point as the appropriate endpoint. Thus a positive half-neighbourhood of $x$ is a set of the form $[x, y]$.

A technical concept we will use is that of $f$-covering. We say of two closed intervals $J$ and $K$ that $J f$-covers $K$ if $f\left(J^{\prime}\right)=K$ for some sub-interval $J^{\prime}$ of $J$. (For maps of the interval, this is equivalent to $f(J) \supseteq K$; for maps of the circle, it is stronger.) The importance of $f$-coverings lies in the fact that if $J f^{n}$-covers itself for some $n$, then $f$ has a periodic point in $J$.

\section{Unstable sets}

The basic tool for proving all three theorems is the analysis of one-sided unstable sets. We review here the basic facts of this theory from [1], [3] and [7]. The definition we adopt is that of [1]; the unstable sets in [3] and [7] are the closures of the ones we consider here.

For a fixed point $p$ of $f$ and a side $S$, the one-sided unstable set of $p$ is

$$
W^{u}(p, f, S)=\bigcap_{U} \bigcup_{k \geq 0} f^{k}(U)
$$

where the intersection is taken over all $S$-half-neighbourhoods of $p$. Each one-sided unstable set is a (possibly degenerate) interval (possibly the whole circle) containing $p$ which is mapped onto itself by $f$. We remind the reader that there are no universal relations between the two one-sided unstable sets. In particular, a one-sided unstable set need not be a half-neighbourhood.

For a fixed point $p$ of $f^{N}$, the unstable sets (under $f^{N}$ ) of the points in the $f$-orbit of $p$ are related in the manner stated in the following lemma, which summarizes the relevant portions of lemmas $8.1-8.3$ of [3]. 
LEMMA 1. Let $p$ be a fixed point of $f^{N}$ and let $S_{0}=+$ or - . If $W^{u}\left(p, f^{N}, S_{0}\right)$ is non-degenerate, then for each $i \geq 0$ there is a side $S_{i}$ at $f^{i}(p)$ such that

(a) for every $S_{0}$-half-neighbourhood $U$ of $p, f^{i}(U)$ contains an $S_{i}$-half-neighbourhood of $f^{i}(p)$.

Write $W_{i}$ in place of $W^{u}\left(f^{i}(p), f^{N}, S_{i}\right)$. Then

(b) $W_{i}=f^{i}\left(W_{0}\right)$

(c) $W_{i+N}=W_{i}$;

(d) if $f^{i}(p) \in$ int $\left(W_{j}\right)$, then $W_{i}=W_{j}$.

Note that (c) does not assert that $S_{i+N}=S_{i}$, but only that the one-sided unstable sets are the same. The proof of (d) may require a moment's thought. When $W_{j}$ is a proper sub-interval of the circle, (d) is just a restatement of lemma 8.3 of [3]. On the other hand, if $W_{j}$ is the whole circle, then (b) implies that $f$ maps the circle onto itself so that $W_{i}$ is the whole circle as well.

To get some feeling for one-sided unstable sets, the reader is invited to verify the lemma for the examples which appear in $\$ 5$.

4. Non-wandering points: theorem $A$

Recall that $x$ is non-wandering under $f$, denoted $x \in \Omega(f)$, if for every neighbourhood $U$ of $x$,

$$
f^{n}(U) \cap U \neq \varnothing \quad \text { for some } n \geq 1 .
$$

The following two technical lemmas are proved using essentially the same arguments used to prove the corresponding results [3] for maps of the interval. For details, see $[6]$.

LEMMA 2. If $x \in \Omega(f)$, then for every neighbourhood $U$ of $x, x \in f^{n}(U)$ for some $n \geq 1$.

LEMMA 3. If $x \in \Omega(f)$ has an infinite orbit, then $x \in \Omega\left(f^{n}\right)$ for every $n$.

It is easy to construct, for any pre-assigned even $n$, a map $f$ with $\Omega\left(f^{n}\right) \neq \Omega(f)-$ just embed the appropriate example from [3] in a map of the circle.

It follows from lemma 3 that to prove theorem $A$ it suffices to prove

(*) If $x \in \Omega(f)$ and the orbit of $x$ contains a fixed point of $f^{N}$, then $x \in \Omega\left(f^{n}\right)$ for every odd $n$.

We do so by induction on $N$. Before we begin the induction we state the following technical lemma, which is an immediate consequence of lemmas 1 and 2 .

LEMMA 4. If $x \in \Omega(f)$ and $f^{k N}(x)=p$ is a fixed point of $f^{N}$, then there are sides $S_{i}(i \geq 0)$, consistent with lemma 1 , such that

(a) for every neighbourhood $U$ of $x, f^{k N+i}(U)$ contains an $S_{i}$-half-neighbourhood of $f^{k N+i}(x)$

(b) $x \in W_{i}=W^{u}\left(f^{i}(p), f^{N}, S_{i}\right) \quad$ for some $i$.

The following lemma proves $(*)$ for $N=1$ and 2 . The proof we give is a streamlined version of the proof of Lemma 9.1 of [3]. 
LEMMA 5. If $x \in \Omega(f)$ and the orbit of $x$ contains a fixed point of $f^{2}$, then $x \in \Omega\left(f^{n}\right)$ for every odd $n$.

Proof. We may assume that $x$ is not itself periodic. Let $f^{2 k}(x)$ be a fixed point of $f^{2}$. Using the notation of lemma 4 , there exists $i=0$ or 1 and a side $S=S_{i}$ of $q=f^{2 k+i}(x)$ such that for every neighbourhood $U$ of $x, f^{2 k+i}(U)$ contains an $S$-half-neighbourhood of $q$ and such that $x \in W^{u}\left(q, f^{2}, S\right)$.

Let $U$ be a neighbourhood of $x$ and let $V$ be an $S$-half-neighbourhood of $q$ contained in $f^{2 k+i}(U)$ with

$$
x \notin V \cup f^{2}(V) \cup f^{4}(V) .
$$

Note that $x \in f^{2 j}(V)$ for some $j \geq 3$. There are three possibilities for the behaviour of $V$ under $f^{2}$ :

(1) $f^{2}(V) \subseteq V$

(2) $f^{2}(V) \supseteq V$;

(3) $f^{2}(V)=V^{\prime} \cup V^{\prime \prime}$, where $V^{\prime}$ is a (possibly degenerate) $S$-half-neighbourhood of $q$ properly contained in $V$ and $V^{\prime \prime}$ is a non-degenerate half-neighbourhood of $q$ on the other side.

If (1) holds, then $x \notin W^{u}\left(q, f^{2}, S\right)$. If (2) holds, then $x \in f^{2 m+2 j}(V)$ for every $m$. If (3) holds, then $f^{2}\left(V \cup V^{\prime \prime}\right)$ is not contained in $V \cup V^{\prime \prime}$, so either

(3a) $f^{2}\left(V^{\prime \prime}\right) \supseteq V^{\prime \prime}$, or

(3b) $f^{2}\left(V^{\prime \prime}\right) \supseteq V$.

If $(3 \mathrm{a})$ holds, then

$$
x \in f^{2(m-1)+2 j}\left(V^{\prime \prime}\right) \subseteq f^{2 m+2 j}(V) \quad \text { for every } m .
$$

If (3b) holds, then $x \in f^{4 m+2 j}(V)$ for every $m$. In all three possible situations - (2), (3a), (3b) - we have $x \in f^{4 m+2 j+2 k+i}(U)$ for every $m$. Thus $\left\{r \mid x \in f^{r}(U)\right\}$ contains all sufficiently large integers in some residue class modulo 4 . But every such class contains arbitrarily large multiples of every odd number. Thus $x \in \Omega\left(f^{n}\right)$ for every odd $n$.

Lemma 6. Suppose $x \in \Omega(f)$ has a finite orbit. If, with notation as in lemma $4, x \in$ int $\left(W_{i}\right)$ for some $i$, then $x \in \overline{\operatorname{Per}(f)}$ and hence $x \in \Omega\left(f^{n}\right)$ for every $n$.

Proof. We again assume that $x$ is not itself periodic. Let $f^{k N}(x)=p$ be a fixed point of $f^{N}$. There are sides $S$ at $x$ and $S_{i}$ at $f^{k N+i}(x)=q$ such that for every $S$-halfneighbourhood $U$ of $x, f^{k N+i}(U)$ contains an $S_{i}$-half-neighbourhood of $q$.

Let $G$ be a lift of $f^{N}$ with a fixed point $Q$ satisfying $\pi(Q)=q$. Let $X$ be such that $\pi(X)=x$ and $Q \in(X-1, X)$. Since $x \in$ int $\left(W^{u}\left(q, f^{N}, S_{i}\right)\right)$ and $x$ is not periodic, at least one of the following must hold:

(1) $X \in \operatorname{int}\left(W^{u}\left(Q, G, S_{i}\right)\right)$;

(2) $X-1 \in$ int $\left(W^{u}\left(Q, G, S_{i}\right)\right)$;

(3) both $X$ and $X-1$ are in $W^{u}\left(Q, G, S_{i}\right)$.

In each case it follows that if $U$ is a small enough $S$-half-neighbourhood of $x$ contained in $W_{i}$, then $U f^{j N+k N+i}$-covers itself for some $j$. Thus every such $U$ contains a periodic point and hence $x \in \overline{\operatorname{Per}(f)}$. 
Now comes the inductive step.

LEMMA 7. Let $N \geq 3$. If $x \in \Omega(f)-\overline{\operatorname{Per}(f)}$ and the orbit of $x$ contains a fixed point of $f^{N}$, then there exist $m \geq 2$ and $M<N$ such that for $g=f^{m}, x \in \Omega(g)-\overline{\operatorname{Per}(g)}$ and the $g$-orbit of $x$ contains a fixed point of $\mathrm{g}^{M}$.

Proof. Let $f^{k N}(x)=p$ be a fixed point of $f^{N}$. Without loss of generality, $N$ is the least period of $p$, for otherwise the conclusion holds with $M=$ the least period of $p$, and $m=N / M$.

Let $W_{i}, i \geq 0$, be given by lemma 4 . Note that if $x \in W_{0}$, then the conclusion holds with $m=N$ and $M=1$. Suppose then that $x \notin W_{0}$ and hence that $x \in W_{i}$ for some $i, 1 \leq i \leq N-1$.

Claim 1. The endpoints of $W_{i}$ are $x$ and $p$.

By lemma $6, x$ must be an endpoint of $W_{i}$. Since $W_{i}$ is $f^{N}$-invariant, $p \in W_{i}$. If $p$ were not an endpoint of $W_{i}$, then by lemma $1, W_{0}=W_{i}$ which contains $x$. This proves claim 1.

Claim 2. $W_{i}$ contains only $p$ and $f^{i}(p)$ from the orbit of $p$.

We use lemma 1 repeatedly. Suppose $f^{j}(p) \in W_{i}$ where $f^{j}(p) \neq p$ and $f^{j}(p) \neq f^{i}(p)$. Then $f^{j}(p) \in$ int $\left(W_{i}\right)$ and hence $W_{i}=W_{j}$. Let $t=|i-j|$. Then $W_{i}$ is $f^{t}$-invariant and hence contains the entire $f^{t}$-orbit of $p$. In particular, for some $r$,

$$
f^{t}(p), f^{2 t}(p), \ldots, f^{r t}(p) \in \text { int }\left(W_{i}\right) \text { and } f^{(r+1) t}(p)=p
$$

Then $W_{i}=W_{r t}$ and hence $W_{0}=f^{t}\left(W_{r t}\right)=W_{i}$ which contains $x$. This proves claim 2 .

Claim 3. Each $W_{j}$ contains exactly two members of the orbit of $p$.

This follows immediately from claim 2 .

We now complete the proof of the lemma. If $N=2 i$, the conclusion follows with $M=2$ and $n=N / 2$. Suppose that $N \neq 2 i$. Then the points $p, f^{i}(p)$, and $f^{2 i}(p)$ are distinct. Now $W_{2 i}$ contains both $f^{i}(p) \in \operatorname{int}\left(W_{i}\right)$ and $f^{2 i}(p) \notin W_{i}$. Thus $p \notin W_{2 i}$ and hence $x \in$ int $\left(W_{2 i}\right)$. Then by lemma $6, x \in \overline{\operatorname{Per}(f)}$.

Assertion (*) and hence theorem A now follow easily from lemmas 5 and 7 . Note that our proof of theorem $\mathrm{A}$ works for maps of the interval as well as for maps of the circle.

\section{Homoclinic points: theorem $B$}

Recall that $x$ is a homoclinic point if for some $N$ there is a fixed point $p$ of $f^{N}$ such that $x \neq p, p$ is in the $f^{N}$-orbit of $x$, and $x \in W^{u}\left(p, f^{N}, S\right)$ for some side $S$. In this case we say that $x$ is homoclinic to $p$. We sometimes call such a point a 'strong' homoclinic point, to distinguish it from a 'weak' homoclinic point, which we define below.

A point $x$ is a weak homoclinic point if for some $N$ there is a fixed point $p$ of $f^{N}$ such that $x \neq p, p$ is in the $f^{N}$-orbit of $x$, and $x \in W^{u}\left(q, f^{N}, S\right)$ for some $q$ in the $f$-orbit of $p$ and some side $S$. It follows from lemma 4 that any point in $\Omega(f)-\operatorname{Per}(f)$ with a finite orbit is a weak homoclinic point. Conversely, a non-wandering weak homoclinic point has a finite orbit but is not periodic. The distinction between strong and weak homoclinic points is illustrated by the following example. 
Example 1. Let $f$ be a map of the circle with the following properties: $f$ has a periodic orbit $\{p, q\}$ of period 2 with $p$ and $q$ diametrically opposite; $f$ maps $[p, q]$ isometrically onto $[q, p]$ preserving orientation; and for some $x \in(q, p), f$ collapses $[q, x]$ to $p$ and uniformly stretches $[x, p]$ onto $[p, q]$ preserving orientation. Then $x$ is a weak homoclinic point but there are no strong homoclinic points.

We note in passing that this example shows that on the circle, unlike the interval [6], [8], [11], the existence of a weak homoclinic point does not imply the existence of a strong homoclinic point.

We say that $f$ has a horseshoe if for some $N$ there are disjoint closed intervals $J$ and $K$ such that each of $J$ and $K f^{N}$-covers both $J$ and $K$. When $f$ has a horseshoe as above, the $f^{N}$-invariant set

$$
H=\bigcap_{i=0}^{\infty} f^{-i N}(J \cup K),
$$

the set of points whose $f^{N}$-orbit lies in $J \cup K$, has the full one-sided shift on two symbols as a continuous factor, via the map that assigns to each point in $H$ its itinerary under $f^{N}$. This factor map takes the periodic points of $f^{N} \mid H$ onto the periodic points of the shift, as a consequence of the fact that any interval which $f^{i}$-covers itself contains a fixed point of $f^{i}$.

The Homoclinic Point Theorem [1], [9], [10] states (in part) that a map of the interval (or the reals) which has a strong homoclinic point also has a horseshoe.

It is easy to construct a map of the circle with a strong homoclinic point but no horseshoes.

Example 2. Let $f$ be a map of the circle with the following properties: $f$ has a fixed point $p, x \neq p, f$ collapses $[x, p]$ to $p$ and homeomorphically stretches $(p, x)$ onto the complement of $p$, with every point moving a positive distance forward. Then $x$ is homoclinic to $p$ but $f$ cannot have a horseshoe, since for any closed interval $J$ which does not contain $p$, and for any $n, f^{n}(J)$ does not contain $J$.

We will prove the two implications of theorem B separately. The easier implication is:

Proposition 1. If $f$ has a horseshoe, then it has a non-wandering homoclinic point.

A preliminary technical observation will streamline our proof. If $J f$-covers $K$, it is clear that we can choose the subinterval $J^{\prime}$ for which $f\left(J^{\prime}\right)=K$ so that its endpoints map onto the endpoints of $K$. We then refer to $J^{\prime}$ as a precise pre-image of $K$ in $J$. If $J^{\prime}=[a, b]$ is a precise pre-image of $K=[c, d]$ then $f$ either preserves the endpoint order $(f(a)=c, f(b)=d)$ or reverses it $(f(a)=d, f(b)=c)$.

Lemma 8. Suppose $J$-covers $K$ and $J^{\prime}$ is a precise pre-image of $K$ in $J$. If $f$ preserves (respectively reverses) the endpoint order on $J^{\prime}$, then every sub-interval $L$ of $K$ has a precise pre-image in $J^{\prime}$ on which fpreserves (respectively reverses) the endpoint order.

We omit the straightforward proof. 
Proof of proposition 1. Suppose $f$ has a horseshoe, exhibited by $J=\left[a_{0}, b_{0}\right], K$, and $f^{N}$. Since a non-wandering point for $f^{N}$ is non-wandering for $f$, we may assume that $N=1$.

In particular, $J f$-covers itself, and hence it $f^{2}$-covers itself as well. A precise pre-image $J^{\prime}$ of $J$ which reverses endpoint order must, by lemma 8 , contain a precise pre-image $J^{\prime \prime}$ of $J^{\prime}$ on which $f$ also reverses endpoint order. But then $f^{2}$ maps $J^{\prime \prime}$ onto $J$ preserving endpoint order. Thus (replacing $f$ by $f^{2}$ if necessary) we can assume that $J_{1}=\left[a_{1}, b_{1}\right]$ is a precise pre-image of $J$ in $J$ on which $f$ preserves endpoint order. Invoking lemma 8 inductively, we find a nested sequence of intervals $J_{m}=\left[a_{m}, b_{m}\right]$ such that $J_{m}$ is a precise pre-image of $J_{m-1}$ in $J_{m-1}$ on which $f$ preserves endpoint order. In particular, we have

$$
a_{m-1}=f\left(a_{m}\right) \leq a_{m}<b_{m} \leq f\left(b_{m}\right)=b_{m-1},
$$

so that the sequences $a_{m}$ and $b_{m}$ converge monotonically to fixed points, say $a$ and $b$, of $f$. Furthermore, given a negative half-neighbourhood $U$ of $a$ and a positive half-neighbourhood $V$ of $b, a_{m} \in U$ and $b_{m} \in V$ for all sufficiently large $m$. This implies that $a_{i} \in W^{u}(a, f,-)$ and $b_{i} \in W^{u}(b, f,+)$ for $i=0,1,2, \ldots$ Now, since $\left[a_{0}, b_{0}\right] f$-covers $K$ and

$$
f\left[a_{1}, b_{1}\right] \cap K=\varnothing,
$$

either $\left[a_{0}, a_{1}\right]$ or $\left[b_{1}, b_{0}\right] f$-covers $K$. Without loss of generality, we assume the latter. Then, since the $f$-invariant set $W^{u}(b, f,+)$ contains $\left[b, b_{0}\right]$, it contains $K$ as well.

On the other hand, $K f$-covers $J$, so there is a point $x \in K$ such that $f(x)=b$ and the image of every neighbourhood of $x$ contains a positive half-neighbourhood of $b$. Then $x$ is non-wandering and homoclinic to $b$.

An examination of the proof of the Homoclinic Point Theorem in [1] reveals that, for maps of the interval or the reals, the intervals exhibiting the horseshoe can be chosen to lie inside any pre-assigned neighbourhood of the periodic point involved. In particular, if some lift $F$ of $f$ has a homoclinic point, then $F$ has a horseshoe exhibited by intervals which are contained in an interval of length less than one, and hence which project under $\pi$ to disjoint intervals on the circle. These latter intervals exhibit a horseshoe for $f$. Thus we have

LEMMA 9. If some lift of $f$ has a homoclinic point, then $f$ has a horseshoe.

We will make use of the following fact, which (for maps of the interval) is implicit in [1] and explicit in [8] and [11].

LEMMA 10. Let $F$ be a map of the interval or reals. If there is a fixed point $P$ of $F$ and a point $X>P$ with $X \in W^{u}(P, F,-)-W^{u}(P, F,+)$, then $F$ has a homoclinic point.

We formulate a strengthened converse of proposition 1 as

PROPOSITION 2. If a map of the circle has a non-periodic non-wandering point with a finite orbit, then it has a horseshoe.

We first prove a special case. 
LEMMA 11. If $x \in \Omega(f)$ is not a fixed point, but has a fixed point in its orbit, then $f$ has a horseshoe.

Proof. We may assume that $f(x)=\pi(0)$ is a fixed point and choose a lift $F$ of $f$ such that $F(0)=0$. By lemma 9 , we may assume that $F$ has no homoclinic points. Let $X$ be the unique point between 0 and 1 such that $\pi(X)=x$. Then either $X$ or $X-1$ belongs to

$$
W^{u}(0, F,+) \cup W^{u}(0, F,-) .
$$

We assume that it is $X$, noting that the proof in the other case is similar. Then by lemma $10, X \in W^{u}(0, F,+)$, for otherwise $F$ has a homoclinic point.

To show that $f$ has a horseshoe, it suffices to show

$$
W^{u}(0, F,+) \text { contains some } Y>1 \text {. }
$$

To see this, note first that we may assume that $Y<2$. There exist $m>0$ and points

$$
0<X_{0}<X_{1}<X_{2}<Y-1
$$

such that

$$
F^{m}\left(X_{2}\right)=Y, \quad F^{m}\left(X_{1}\right)=1 \quad \text { and } \quad F^{m}\left(X_{0}\right)=X_{2} .
$$

Then the intervals $\pi\left[0, X_{0}\right]$ and $\pi\left[X_{1}, X_{2}\right]$ are disjoint and each $f^{m}$-covers both.

To prove (*), note that since $f(x)=\pi(0)$ is a fixed point, $F(X)$ must be an integer, which is non-zero since otherwise $X$ is homoclinic to 0 . Furthermore, if $F(X)>1$, then (*) holds with $Y=F(X)$. Thus we have two cases: $F(X)=1$ and $F(X)<0$.

Case 1. $F(X)=1$.

We may assume that $F(1)=1$; for if $F(1)<0$, then some point in $(X, 1)$ is homoclinic to 0 ; if $F(1)=0$, then 1 is homoclinic to 0 ; and if $F(1)>1$, then (*) holds with $Y=F(1)$. Thus we have $F(X)=F(1)=1$. For every neighbourhood $U$ of $X, F(U)$ contains at least a half-neighbourhood of 1 , otherwise $x \notin \Omega(f)$. In fact, it contains a positive half-neighbourhood, since if $F(U)$ is a negative half-neighbourhood of 1 , then by lemma $2, X \in W^{u}(1, F,-)$, making $X$ homoclinic to 1 . But since $F(U)$ contains a positive half-neighbourhood of $1,(*)$ holds for some $Y \in F(U)$.

Case 2. $F(X)<0$.

If $F^{2}(X)<0$ as well, then $\operatorname{deg}(f)>0$ and hence $F^{m}(X)<0$ for all $m \geq 1$. Now $F[0, X]$ contains no point to the right of 0 , since otherwise some point in $[0, X]$ is homoclinic to 0 . Similarly, for all $m \geq 1, F^{m}[0, X]$ contains no point to the right of 0 and hence $X \notin W^{u}(0, F,+)$.

Suppose then that $F^{2}(X) \geq 0$. If $F^{2}(X)=0$, then $X$ is homoclinic to 0 . If $F^{2}(X)=$ 1 , then $F(X)=-1$, so $\operatorname{deg}(f)=-1$ and hence $F(X-1)=0$, making $X-1$ homoclinic to 0 . This leaves only $F^{2}(X)>1$, and so $(*)$ holds with $Y=F^{2}(X)$. This proves (*) and hence the lemma.

Proof of proposition 2. Let $x \in \Omega(f)$ be non-periodic and suppose $f^{k N}(x)=p$ is a fixed point of $f^{N}$. If $x \in \overline{\operatorname{Per}(f)}$, then $x \in \Omega\left(f^{N}\right)$. By lemma $11, f^{N}$ has a horseshoe and hence so does $f$.

Suppose then that $x \notin \overline{\operatorname{Per}(f)}$. Using the notation of lemma 4, we have $x \in W_{i}$ for some $i, 0 \leq i \leq N-1$. If $x \in W_{0}$, then $x \in \Omega\left(f^{N}\right)$ and $f$ has a horseshoe as in the 
preceding paragraph. Suppose then that $x \notin W_{0}$ and $x \in W_{i}$ where $1 \leq i \leq N-1$. As in the proof of lemma 7, the endpoints of $W_{i}$ are $x$ and $p$. Thus $W_{i}$ is a compact, $f^{N}$-invariant, proper sub-interval of the circle. Let $q=f^{i}(p)$ and suppose without loss of generality that $S_{i}=+$ and hence that $W_{i}=W^{u}\left(q, f^{N},+\right)$.

If $W_{i}=[p, x]$, then $f^{j N}(y)=x$ for some $y \in(q, x)$ and some $j \geqslant k$. Since $f^{j N}(x)=p$, there exists $z \in(y, x)$ such that $f^{j N}(z)=q$. But then $z$ is a homoclinic point for $f^{N} \mid W_{i}$. By the Homoclinic Point Theorem, $f^{N} \mid W_{i}$ has a horseshoe, and hence $f$ has one as well.

If $W_{i}=[x, p]$, then by lemma 10 , either $f^{N} \mid W_{i}$ has a homoclinic point or $x \in$ $W^{u}\left(q, f^{N} \mid W_{i},-\right)$. In either case, as in the preceding paragraph, $f$ has a horseshoe.

Note that we have proved a stronger version of theorem $B$, analogous to the proposition in [8].

THEOREM B+. For a continuous map $f$ of the circle, the following are equivalent:

(1) f has a horseshoe;

(2) f has a non-wandering (strong) homoclinic point;

(3a) f has a non-wandering weak homoclinic point;

(3b) $f$ has a non-periodic non-wandering point with a finite orbit.

We remark that these conditions are also equivalent to each of the following:

(4) f has positive topological entropy;

(5) $f$ has periodic points with least periods $n<m$ where $m / n$ is not a power of 2 .

(1) implies (5) follows from the fact that the factor map from the horseshoe preserves periods, (5) implies (4) from [2], and (4) implies (1) from [5].

\section{Maps with closed periodic set: theorem $C$}

Our proof of theorem $\mathrm{C}$ will follow from an analysis of the non-wandering set for maps with no horseshoes. That this is the right situation to look at follows from

Lemma 12. If $f$ has a horseshoe, then $\operatorname{Per}(f)$ is not closed.

Proof. Recall from the earlier discussion of horseshoes that for some $N$ there is a compact $f^{N}$-invariant set $H$ such that $f^{N} \mid H$ has the full one-sided shift on two symbols as a continuous factor. Furthermore, $\operatorname{Per}\left(f^{N} \mid H\right)$ maps onto the set of periodic points of the shift. If $\operatorname{Per}(f)$ is closed, then so is $\operatorname{Per}\left(f^{N} \mid H\right)$ and hence also the set of periodic points of the shift. But this last set is not closed.

LEMMA 13. If for some lift $F$ of $f$, there is an interval $J \subseteq[0,1]$ of length less than one such that for some $m \geq 1, F^{m}(J)$ contains three consecutive integers, then $f$ has a horseshoe.

The proof of lemma 13 is straightforward (see lemma 5.10 of [6]).

Lemmas 12 and 13 allow us to concentrate on maps of degree 0 or \pm 1 . We handle these cases separately. 
Proposition 3. Suppose $f$ has degree zero and $F$ is a lift of $f$. We have

(1) $\pi[\Omega(F)]=\Omega(f)$;

(2) if $\operatorname{Per}(f)$ is closed, then so is $\operatorname{Per}(F)$.

Proof. Since the range of $F$ is compact, $F$ has a fixed point, and so we can assume without loss of generality that $F(0)=0$. Let $I$ be a compact interval which contains the range of $F$ and has (distinct) integer endpoints. Then $\Omega(F)=\Omega(F \mid I)$ and $x \mid I$ is a finite-to-one factor map of $F \mid I$ onto $I$. We will abuse notation slightly by using $F$ in place of $F \mid I$ and $\pi$ in place of $\pi \mid I$.

To show (1), we need only show that $\Omega(f) \subseteq \pi[\Omega(F)]$. Given $x \in \Omega(f)$, we may assume that $0 \notin \pi^{-1}(x)$, since otherwise $x \in \pi[\Omega(F)]$. Let

$$
\pi^{-1}(x)=\left\{X^{(1)}, \ldots, X^{(m)}\right\}
$$

observe that $X^{(j)} \in$ int $(I)$ for all $j$. By lemma 2 , there exist $x_{i} \rightarrow x$ and $n_{i} \geq 1$ such that $f^{n_{i}}\left(x_{i}\right)=x$. We may assume that $0 \notin \pi^{-1}\left(x_{i}\right)$ and hence that each $\pi^{-1}\left(x_{i}\right)$ consists of exactly $m$ points in $I$,

$$
\pi^{-1}\left(x_{i}\right)=\left\{X_{i}^{(1)}, \ldots, X_{i}^{(m)}\right\} .
$$

We can label these points so that $X_{i}^{(j)} \rightarrow X^{(j)}$ for each $j$. Note that $F\left(X_{i}^{(j)}\right)$ depends on $i$ but not on $j$, since $f$ has degree zero.

Now $\pi\left[F^{n_{i}}\left(X_{i}^{(j)}\right)\right]=x$ for all $i$ and all $j$, and $\pi^{-1}(x)$ is finite while $F^{n_{i}}\left(X_{i}^{(j)}\right)$ is independent of $j$. It follows that for some $k$,

$$
F^{n_{i}}\left(X_{i}^{(j)}\right)=X^{(k)}
$$

for infinitely many $i$ and all $j$, and hence that

$$
X^{(k)} \in \Omega(F) \cap \pi^{-1}(x) .
$$

This proves (1).

To show (2), suppose $X \in \overline{\operatorname{Per}(F)}-\operatorname{Per}(F)$. Then $\pi(X) \in \overline{\operatorname{Per}(f)}=\operatorname{Per}(f)$. Thus $\pi(X)$ has a finite orbit and so $X$ does too. This makes $X$ a weak homoclinic point. Then $F$ must have a strong homoclinic point as well (see [6], [8] or [11]). Hence by lemma $9, f$ has a horseshoe and then by lemma $12 \operatorname{Per}(f)$ is not closed.

To obtain the analogue of proposition 3 for maps of degree one we need to assume more.

Proposition 4. Suppose $f$ is a map of degree one which has no horseshoes. If $F$ is a lift of $f$ which has a fixed point, then

(1) $\operatorname{Per}(F)=\pi^{-1}[\operatorname{Per}(f)]$;

(2) $\Omega(F)=\pi^{-1}[\Omega(f)]$.

Proof. We may assume that 0 is a fixed point of $F$. We first establish

$$
X-1<F^{n}(X)<X+1 \quad \text { for all } X \text { and all } n \text {. }
$$

Since $\operatorname{deg}(f)=1, F^{n}(X+k)=F^{n}(X)+k$, so we need prove $(*)$ only for $X \in(0,1)$. If $F^{n}(X) \geq X+1$, then

$$
F^{2 n}[0, X] \supseteq F^{n}[0, X+1] \supseteq[0, X+2],
$$


and so by lemma $13 f$ has a horseshoe. Similarly, if $F^{n}(X) \leq X-1$, then

$$
F^{2 n}[X, 1] \supseteq[X-2,1],
$$

and again $f$ has a horseshoe. This proves (*).

To prove (1), it suffices to show that if $\pi(X) \in \operatorname{Per}(f)$, then $X \in \operatorname{Per}(F)$. If $\pi(X) \in \operatorname{Per}(f)$, say $f^{n}(\pi(X))=\pi(X)$, then $F^{n}(X)=X+k$ for some integer $k$. By $(*), k=0$ and hence $X \in \operatorname{Per}(F)$.

To prove (2), it suffices to show that if $x=\pi(X) \in \Omega(f)$, then $X \in \Omega(F)$. Since $x \in \Omega(f)$, lemma 2 implies that there exist $x_{i} \rightarrow x$ and $n_{i} \geq 1$ such that $f^{n_{i}}\left(x_{i}\right)=x$. Let $X_{i}$ be the point closest to $X$ in $\pi^{-1}\left(x_{i}\right)$. Then $X_{i} \rightarrow X$ and $F^{n_{i}}\left(X_{i}\right)=X+k_{i}$ for some integer $k_{i}$. The convergence of $X_{i}$ to $X$ together with $(*)$ imply that for $i$ sufficiently large, $k_{i}=-1,0$, or 1 . Thus, for a subsequence of $X_{i}$ (which we still denote $X_{i}$ ) $F^{n_{i}}\left(X_{i}\right)$ is constant and equals $X-1, X$, or $X+1$.

Suppose $F^{n_{i}}\left(X_{i}\right)=X+1$. If $F(X)<X$, then for some $\delta>0$ and all sufficiently large $i, F\left(X_{i}\right)<X-\delta$. Using (*) again, for these $i$ we have

$$
F^{n_{i}}\left(X_{i}\right)=F^{n_{i}-1}\left(F\left(X_{i}\right)\right)<F\left(X_{i}\right)+1<X+1-\delta,
$$

contradicting the assumption that $F^{n_{i}}\left(X_{i}\right)=X+1$. Using the fact that

$$
F^{n_{i}+1}\left(X_{i}\right)=F(X)+1 \text {, }
$$

similar arguments lead to a contradiction when $F(X)>X$. Hence $F(X)=X$ and $X \in \Omega(F)$.

In the same way, if $F^{n_{i}}\left(X_{i}\right)=X-1$, then $F(X)=X$ and' $X \in \Omega(F)$. Finally, if $F^{n_{i}}\left(X_{i}\right)=X$, then $X \in \Omega(F)$ by definition.

We now assemble a proof of theorem C. Suppose Per $(f)$ is closed and non-empty. By lemma 12, $f$ has no horseshoes. It follows from lemma 13 that $\operatorname{deg}(f)=0$ or \pm 1 . If $\operatorname{deg}(f)=0$, then by proposition 3 , for a lift $F$ of $f, \operatorname{Per}(F)$ is closed and non-empty. Then by $[8], \Omega(F)=\operatorname{Per}(F)$, and by proposition 3 again,

$$
\Omega(f)=\pi[\operatorname{Per}(F)]=\operatorname{Per}(f) .
$$

If $\operatorname{deg}(f)= \pm 1$ and $x \in \Omega(f)-\operatorname{Per}(f)$, then $x$ must have an infinite orbit, since otherwise by theorem B $f$ has a horseshoe. But then by lemma $3, x \in \Omega\left(f^{n}\right)$ for all $n$. Choose $n$ even (so that $f^{n}$ has degree one) and such that $f^{n}$ has a fixed point. Let $G$ be a lift of $f^{n}$ which has a fixed point. Since $f^{n}$ has no horseshoes, it follows from proposition 4 that $\operatorname{Per}(G)$ is closed and non-empty, and hence by [8] again that $\Omega(G)=\operatorname{Per}(G)$. But then

$$
x \in \Omega\left(f^{n}\right)=\pi[\Omega(G)]=\pi[\operatorname{Per}(G)]=\operatorname{Per}\left(f^{n}\right)=\operatorname{Per}(f) .
$$

The proof is complete.

\section{REFERENCES}

[1] L. Block. Homoclinic points of mappings of the interval. Proc. Amer. Math. Soc. 72 (1978), 576-580.

[2] L. Block, E. M. Coven \& Z. Nitecki. Minimizing topological entropy for maps of the circle. Ergod. Th. \& Dynam. Sys. 1 (1981), 145-149.

[3] E. M. Coven \& Z. Nitecki. Non-wandering sets of the powers of maps of the interval. Ergod. Th. \& Dynam. Sys. 1 (1981), 9-31. 
[4] V. V. Fedorenko \& A. N. Sarkovskii. Continuous mappings of the interval with closed sets of periodic points. In Introduction to Differential and Differential-difference Equations, pp. 137-145. Kiev, 1980. (Russian)

[5] M. Misiurewicz. Horseshoes for mappings of the interval. Bull. Acad. Polon. Sci. Ser. Sci. Math. 27 (1979), 167-169.

[6] I. Mulvey. Periodic, recurrent and non-wandering points for continuous maps of the circle. Ph.D. thesis. Wesleyan University, Middletown, Conn. (1982).

[7] Z. Nitecki. Topological dynamics on the interval. In Ergodic Theory and Dynamical Systems II, pp. 1-73. College Park, Md., 1979-80, Progr. Math. vol. 21. Birkhaüser: Boston, 1982.

[8] Z. Nitecki. Maps of the interval with closed periodic set. Proc. Amer. Math. Soc. 85 (1982), 451-456.

[9] A. N. Sarkovskii. On cycles and structure of continuous mappings. Ukrain. Mat. Z. 17 (1965), 104-111. (Russian)

[10] A. N. Sarkovskii. On the problem of isomorphism of dynamical systems. In Proceedings of the International Conference on Nonlinear Trajectories, vol. 2, pp. 541-545. Kiev, 1970. (Russian)

[11] J.-C. Xiong. Continuous self-maps of the closed interval whose periodic points form a closed set. J. China University of Science and Technology 11 (1981), 14-23. 